\title{
Dr. Ramón Ruiz Maldonado (1937-2017)
}

El cruzar en nuestra existencia con un ser humano como el Dr. Ramón Ruiz Maldonado, hombre culto, sensato, ingenioso y maestro en toda la extensión de la palabra, ilumina y deja huella. Cuando llega el momento de su partida, por una enfermedad prolongada que minó su cuerpo y terminó por encarcelar su mente, la sensación de vacío y nostalgia es inevitable; sólo puede verse hasta cierto punto aliviada por un profundo agradecimiento, ya que aún sin estar presente, su esencia vive en nuestro corazón.

Producto de ese agradecimiento es que lo queremos no sólo recordar, sino lograr que las generaciones más recientes conozcan a través de estas líneas, no sólo a un dermatólogo pediatra excepcional, sino al ser humano... amante de la buena comida, el buen vino y la buena compañía; políglota, poeta y escritor, y por sobre todo, hombre generoso y compartido (Figura 1).

El Dr. Ramón Ruiz Maldonado nació el 7 de noviembre de 1937, en la Ciudad de Puebla, donde cursó los estudios básicos. Desde su niñez sintió inclinación hacia la medicina, probablemente influenciado por la figura de su padrino, el Dr. Francisco Casas, un médico general admirado y querido por él y por sus padres.

En 1957 ingresó a la Facultad de Medicina de la Universidad Nacional Autónoma de México (UNAM), al recién formado "grupo piloto". Al terminar sus estudios, fue seleccionado para realizar el internado rotatorio en el Hospital Foch en París, donde para sobrevivir y aprender el idioma trabajó en la Asociación de Estudiantes de Medicina. A su regreso a México realizó el Servicio Social en el Hospital Central del Instituto Mexicano del Seguro Social en Acapulco, Guerrero.

Se especializó en Dermatología con el Profesor Fernando Latapí en el Hospital General de México y el Centro Dermatológico Dr. Ladislao de la Pascua. Posteriormente, realizaría estudios de posgrado en Dermatología, Micología y Dermatopatología, becado por los gobiernos de diversos países europeos y de los Estados Unidos con los profesores más reconocidos: Robert Degos y Jean Civatte en el Hospital San Luis de París; François Mariat, Gabriel Segretain y Edouard Drouhet en el Instituto Pasteur de París; Albert Wiedmann en el Hospital General de Viena; Jiri Trapl en la Universidad de Carlos en Praga y Hermann Pinkus en la Universidad de Wayne en Detroit.

Durante su "exilio académico" su formación no fue sólo dermatológica, también cultivó los idiomas francés, inglés, alemán e italiano, escribió poesía y una novela corta titulada: Sábado por la tarde que fue premiada en el X Certamen Literario de "La Hora XXV" y publicada en 1967; de la cual, por cierto, aún se pueden conseguir pocos ejemplares usados. 


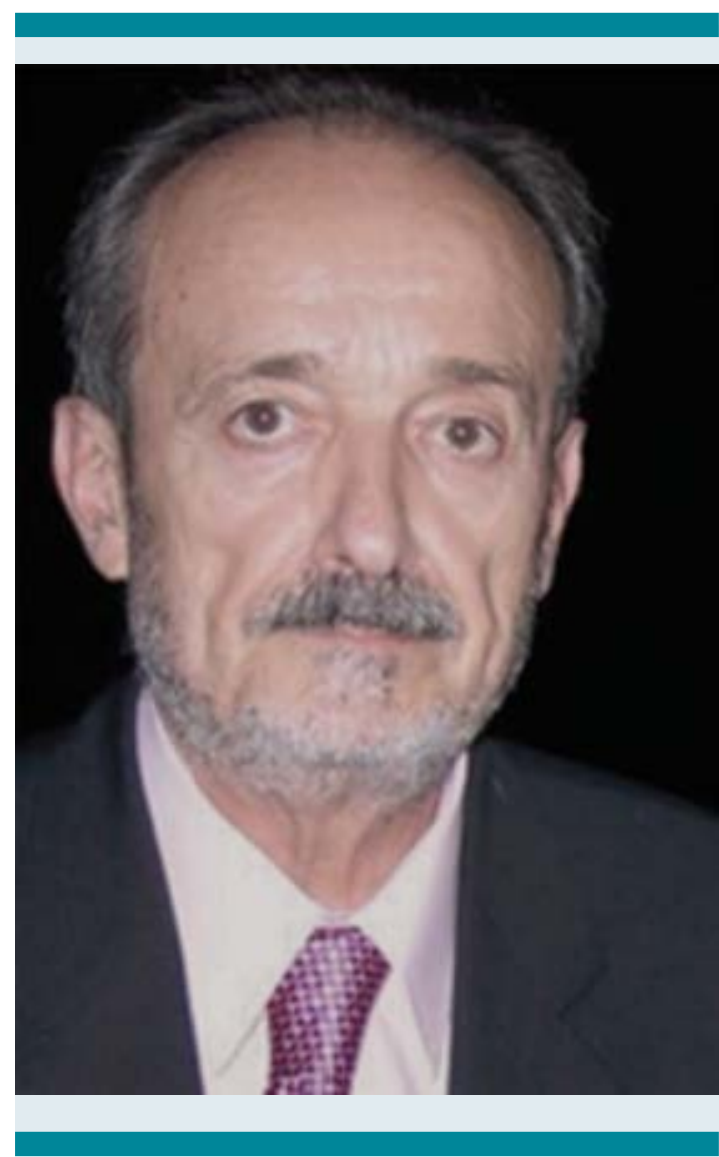

Figura 1. "Extrañar no es estar vacío, sino estar lleno de alguien que se hace presente a pesar de la ausencia".

Alguna vez, durante sus últimos momentos en el Servicio de Dermatología, pocas afortunadas tuvimos la oportunidad de leer su poesía (en hojas ya gastadas por el tiempo, con tinta casi apenas perceptible y con su letra aún impoluta por la enfermedad) y conocer a un hombre romántico al extremo y enamorado del amor.

A su regreso de Europa trabajó como encargado del Laboratorio de Patología del Centro Dermatológico Pascua, entonces dirigido por el Profesor Fernado Latapí, y un año más tarde fue invitado por el Dr. Rigoberto Aguilar Pico recién designado Director del Hospital Infantil de México, como dermatólogo del Servicio de Alergia y Dermatología.

Al inaugurarse en 1970 el Hospital del Niño IMAN, hoy Instituto Nacional de Pediatría (INP), fue invitado por el Dr. Lázaro Benavides Vázquez como Jefe del Servicio de Dermatología y Alergia, que al año siguiente se convertiría en 2 servicios diferentes: el de Alergia con el Dr. José Huerta y el de Dermatología bajo su jefatura, cargo que ocupó hasta 1992, en que fue sucedido por la Dra. Lourdes Tamayo Sánchez, con quién formó una excelente mancuerna.

En 1992 inició como Investigador Titular " $\mathrm{C}$ " de los Institutos Nacionales de Salud, y ascendería en la escala hasta convertirse en Investigador Emérito en el año 2007.

Por otro lado, el Dr. Ruiz Maldonado, ingresó como Académico de la Academia Nacional de Medicina en 1983 donde en algún punto se desempeñó como Coordinador del Área de Dermatología del Departamento de Medicina; y en 1989 como Investigador del Sistema Nacional de Investigadores, en el cual desde 1999 y hasta su deceso fue Investigador Nacional Nivel III.

Las labores de docencia, asistencia e investigación fueron una constante durante toda su vida profesional, e impulsaron el conocimiento y la difusión de la Dermatología Pediátrica en México y en el mundo, pues siempre fue un Profesor comprometido con compartir sus conocimientos a todo aquel que se le acercara.

El Dr. Ruiz Maldonado inició la subespecialidad en Dermatología Pediátrica en México y en numerosos países de Latinoamérica, pues desde 1972 y hasta 1995, el único curso formal de especialización en Dermatología Pediátrica en el mundo fue el del INP. En él, se han formado más de 100 dermatólogos pediatras, con especialización previa en Pediatría o en Dermatología 
General, muchos de los cuales actualmente fungen como jefes o adscritos en diversos servicios de Dermatología Pediátrica latinoamericanos.

En 1989 el Dr. Ruiz Maldonado logró el reconocimiento de la Dermatología Pediátrica como una rama importante de la Dermatología General por la UNAM, y tiempo después por el Consejo Mexicano de Dermatología, que lo nombró Presidente del Consejo Mexicano de Dermatología Pediátrica.

En el ámbito de la docencia se desempeñó como Profesor titular definitivo de Dermatología de la UNAM; Profesor titular del Curso de Especialización en Dermatología Pediátrica del INP; Profesor titular del Curso de Especialización y del Diplomado/Curso Avanzado en Dermatología Pediátrica de la UNAM; Profesor titular del Curso de Posgrado para Médicos Especialistas: "Inmunología y Genética en Dermatología Pediátrica" de la UNAM, y Asesor académico del programa de Maestría y Doctorado en Ciencias Médicas, Odontológicas y de la Salud de la UNAM. Así mismo, dirigió 77 tesis para la obtención de títulos de Pediatría, Dermatología Pediátrica y/o Maestría en Ciencias Médicas.

Para quiénes nos formamos con él, ser alumno del Profesor Ruiz Maldonado ha sido una fortuna y una delicia. Sus aportaciones en las clases que coordinaba, desde aspectos sencillos como la pronunciación adecuada del nombre de algún autor o de una enfermedad hasta su experiencia documentada y forma de tratar alguna patología dermatológica, son invaluables. Su coordinación en días de consulta externa, una oportunidad única de aprender hombro con hombro bajo su tutela.

Entrar a su oficina a hacer alguna pregunta o a presentar algún paciente era todo un ritual. Siempre metido en los libros y escribiendo o revisando artículos, simplemente alzaba la vista y ambas cejas, preguntaba qué se ofrecía e inmediatamente revisaba al paciente, y si era necesario en presencia del paciente consultaba textos y nunca se olvidaba de tomar una adecuada iconografía de la patología del paciente en cuestión.

Su memoria era privilegiada. Era capaz de sacar "del cajón de las dermatosis sin diagnóstico" una fotografía de un caso visto varios años atrás y decir sin más: "esto ya lo habíamos visto" y, a partir de ahí, seguir todo un método de investigación clínica hasta lograr la descripción de una patología nueva.

De personalidad imponente, pero con un corazón más grande aún que su genialidad, impulsó a un gran número de sus alumnos a escribir, investigar e ingresar en sistemas reconocidos de investigación y academia, y a formar servicios de Dermatología Pediátrica en otros lugares de México y Latinoamérica.

Favoreció un ambiente de armonía entre los integrantes del servicio, médicos y residentes. Con mucha frecuencia ofrecía su casa para realizar reuniones, ya fueran de bienvenida o despedida de los residentes o incluso alguna posada. $Y$ en caso de no ser su casa el sitio de reunión, igualmente asistía a todos los eventos.

Ante su genuina preocupación por el bienestar de los pacientes, en especial el de los niños, fue nombrado miembro del Comité Internacional de Dermatología, cuyos doce miembros representaban a la Dermatología internacional ante la Organización Mundial de la Salud. Fue también designado Secretario General de la Fundación Internacional de Dermatología, organización dedicada en primera instancia a la enseñanza de la dermatología en países en desarrollo. El proyecto más ambicioso de la fundación fue la creación del Centro Regional de Entrenamiento en Dermatología en Moshi, 
Tanzania. En dicho centro se han graduado más de cien "Oficiales Médicos en Dermatología" de trece países africanos, que no contaban con dermatólogos para el manejo de enfermedades cutáneas endémicas. El Profesor Ruiz Maldonado acudió durante 15 años consecutivos, una vez por año, a impartir el curso de Dermatología Pediátrica Tropical.

Su afán por compartir el conocimiento llevaron al Dr. Ruiz Maldonado a publicar el primer libro de texto de Dermatología Pediátrica en inglés Ilamado: "Text book of Pediatric Dermatology", a lo que seguirían 5 obras más. Escribió 364 artículos -más de 150 de ellos en revistas indexadas-, 107 capítulos de libros y dictó 774 conferencias en congresos y cursos, tanto nacionales como extranjeros.

Organizó el Primer Simposio, y el Primer (1973) y Noveno (2001) Congresos Internacionales de Dermatología Pediátrica, siendo presidente en ambos. Fundó la Sociedad Internacional de Dermatología Pediátrica; el Colegio Mexicano de Dermatología Pediátrica; la Sociedad Latinoamericana de Dermatología Pediátrica y la Fundación Mexicana para la Dermatología Pediátrica A.C., de las cuales también fue Presidente en diversos periodos. El Dr. Ruiz Maldonado ocupó diversos puestos en varias sociedades y asociaciones dermatológicas de gran prestigio como: la Sociedad Mexicana de Dermatología (Presidente); la Sociedad Internacional de Dermatología
Tropical (Vicepresidente); la sección de Dermatología de la Asociación Médica Panamericana (Vicepresidente); la Fundación Internacional de Dermatología (Secretario General) y la Sociedad Internacional de Dermatología (Presidente).

El Dr. Ruiz Maldonado fue ampliamente conocido y reconocido a nivel internacional a través de nombramientos como miembro honorario o correspondiente en 44 sociedades dermatológicas de gran prestigio en todo el mundo. Por su trayectoria profesional, recibió 36 distinciones en México y en el extranjero, dentro de las que se cuentan: la Medalla de Oro al Mérito otorgada por la Sociedad Latinoamericana de Dermatología Pediátrica (2003), el reconocimiento como Maestro de la Dermatología Iberolatinoamericana (2003) y la Medalla de Oro Alfred Marchionini por la Liga Internacional de Sociedades Dermatológicas (2007).

Nadie más afortunado que su familia, sus amigos, sus pacientes y nosotros, sus alumnos, por haber coincidido en tiempo y espacio con un hombre genial.

Dra. María del Mar Sáez de Ocariz Dra. Lourdes Carola Durán McKinster Dra. María de la Luz Orozco Covarrubias Dra. Carolina Guadalupe Palacios López Dra. María Teresa García Romero Servicio de Dermatología Instituto Nacional de Pediatría 